\title{
JOT JOURNAL OF TURBULENCE
}

http://jot.iop.org/

\section{Effective visualization of stereo particle image velocimetry vector fields of a turbulent boundary layer}

\author{
E K Longmire ${ }^{1,3}$, B Ganapathisubramani ${ }^{1}$, I Marusic ${ }^{1}$, \\ T Urness ${ }^{2}$ and $\mathrm{V}$ Interrante ${ }^{2}$ \\ ${ }^{1}$ Department of Aerospace Engineering and Mechanics, University of \\ Minnesota, Minneapolis, MN 55455, USA \\ 2 Department of Computer Science, University of Minnesota, Minneapolis, \\ MN 55455, USA \\ E-mail: ellen@aem.umn.edu (E K Longmire)
}

Received 18 February 2003

Published 19 June 2003

\begin{abstract}
Stereo particle image velocimetry datasets contain three-dimensional information over a plane, from which multiple quantities can be derived at each point. The task of visualizing these different parameters simultaneously is challenging, and this inhibits our ability to analyse and derive firm conclusions about the physics of the flow. Currently, the common approach is to view each parameter separately in different images. Such an approach is very inefficient, especially for large fields of view where many important structures and features co-exist. In this paper we discuss several ways in which the primary quantities can be viewed simultaneously in the same image. The simplest method is to use different colours for each parameter and to overlay all the different colours on one image. The limitations of such an approach will be described. Other methods considered involve using texture generated from a line integral convolution algorithm to convey instantaneous velocity direction and magnitude. Animated texture is also described, together with variants involving combined colour and out-of-plane height. The use of height in tandem with colour and animated texture is a useful method in distinguishing the different parameters in the regions of overlap.
\end{abstract}

PACS numbers: 47.27.Nz, 47.27.Jv

3 Author to whom any correspondence should be addressed. 


\section{Contents}

1 Introduction $\quad 2$

2 Experimental facility and methods 3

3 Visualization of stereo PIV datasets $\quad 4$

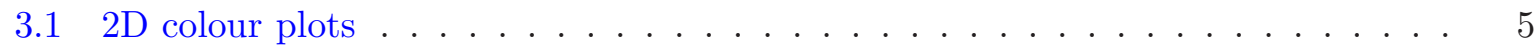

3.2 Line integral convolution of vector fields . . . . . . . . . . . . . . . . . . 6

3.3 Animations with 2D LIC images . . . . . . . . . . . . . . . . . . . . . 9

4 Multi-dimensional visualization 10

5 Summary and conclusions $r$

$\begin{array}{lr}\text { Acknowledgments } & 13\end{array}$

$\begin{array}{ll}\text { References } & 13\end{array}$

\section{Introduction}

The study of coherent structures in turbulent boundary layers has been an active area of research for some time. Much of this work has involved single- or two-point hot-wire measurements and flow visualization. More recently, extensive quantitative data have become available through particle image velocimetry (PIV) and direct numerical simulation (DNS), and thus the need for new analysis and visualization tools has become apparent.

The early work on structure identification in boundary layers goes back to flow visualizations first reported in the 1950s. Elongated streaky structures were observed in the near-wall region of a turbulent boundary layer by Hama [1,2], but were not investigated seriously until the late 1950s [3]. Flow visualization studies that followed concentrated largely on the near-wall region $[4,5]$ and described regions of sweeps $(u>0, w<0)$ and ejections $(u<0, w>0)$ (bursts) that were considered the major source of turbulence production. (Here, $u, v$ and $w$ are the fluctuating velocity components in the streamwise $x$, spanwise $y$ and wall-normal $z$ directions respectively.) The literature on the existence and role of coherent structures in wall turbulence has become rather exhaustive, as has the number of reviews. However, a good overview can be found in the reviews by Cantwell [6], Robinson [7] and Panton [8]. The largest proportion of recent articles have made use of DNS datasets (for example, [9]-[11] etc and articles in [8]) and have concentrated on the viscous near-wall region, and the self-sustaining mechanisms in wall turbulence. Studies of the outer region of the flow commonly cite the importance and existence of hairpin type vortices, similar to those first proposed by Theodorsen [12]. The most comprehensive of these studies was that of Head and Bandyopadhyay [13], who conducted flow visualization studies using smoke in a wind tunnel with laser sheet illumination. These visualizations motivated Perry and Chong [14] to propose a hairpin vortex model for the outer region based on Townsend's [15] attached eddy hypothesis. Later extensions of this vortex model [16] have been shown to describe the second-order statistics in turbulent boundary layers rather well.

A recent extension of the hairpin vortex paradigm has been reported by Adrian et al [17] who performed PIV experiments in streamwise-wall-normal planes and found heads of hairpins organized coherently into packets. They concluded that these spatially organized packets of hairpins explained the long tails in previous correlation measurements, as well as the existence 
of long low speed streaks, sweeps and ejections. Additional PIV measurements by Tomkins and Adrian [18], and studies of DNS of turbulent channel flow by Zhou et al [19] and Liu and Adrian [20], support these arguments. Tomkins and Adrian [18] also found that the spanwise width of the low speed streaks increases away from the wall and the spanwise streak spacing away from the wall is different from that of near-wall streaks. Studies by Christensen and Adrian [21] using linear stochastic estimation techniques on PIV data, and Marusic [22] from attached eddy model calculations, concluded that packets of hairpin vortices are statistically significant and a dominant mechanism for the transport of momentum in the logarithmic region. Ganapathisubramani et al [23] analysed stereo-PIV datasets in streamwise-spanwise planes, finding evidence for the existence of hairpin packets and also concluding that these packets carry a significant proportion of the total Reynolds shear stress even though they occupy only a small percentage of the total area.

The above recent studies are the motivation for this paper. Clearly, a great wealth of information can be gained from PIV and stereo-PIV datasets, and progress has been made. However, what has also become apparent is the difficulty involved in extracting important information from these datasets, and the lack of adequate tools that are available to efficiently analyse them. In this paper, we discuss different ways in which PIV velocity vector fields (or any other 2D flow fields) and related derived quantities can be visualized simultaneously. In particular, we want to find effective ways to visualize stereo-PIV datasets that have 3D information at every point. The availability of $3 \mathrm{D}$ information makes it exceedingly difficult to visualize multiple parameters in a plane. For example, to identify vortex packets and understand their contribution to turbulence dynamics, we need to look at different quantities like vorticity, swirl strength, momentum and Reynolds shear stress (which have components in all three principal directions) simultaneously and we would like to develop a scheme where these parameters can be viewed and studied at the same time instead of separately to make deductions easier.

In the remainder of the paper, we will first present the details of the experiment from which the stereo-PIV data were obtained. Following this, the discussion will focus on one stereoPIV field and the vortex packet scenario. Some novel visualization concepts using line integral convolution (LIC) will be described and discussed.

\section{Experimental facility and methods}

The measurements were carried out in a suction wind tunnel with a working section of $0.33 \mathrm{~m}$ height, $1.22 \mathrm{~m}$ width and $4.8 \mathrm{~m}$ length, and datasets were obtained in streamwise-spanwise $(x-y)$ planes. The coordinate system used is depicted in figure 1. Measurement planes were located $3.3 \mathrm{~m}$ downstream of a trip wire in a zero-pressure-gradient flow with freestream velocity $U_{\infty}=5.9 \mathrm{~m} \mathrm{~s}^{-1}$. Hot wire measurements showed that the turbulence intensity in the freestream was less than $0.2 \%$. The wall shear stress $\left(\tau_{w}\right)$ was computed using the mean velocity profile and the Clauser chart method. All quantities measured and computed are normalized using the skin friction velocity $U_{\tau}\left(=\sqrt{\tau_{w} / \rho}\right.$, where $\rho$ is the density of the fluid $)$ and $\nu$ the kinematic viscosity, and are denoted with a superscript + . The Reynolds number based on the momentum thickness $R e_{\theta}$ was 2500, and $R e_{\tau}=\delta U_{\tau} / \nu$ was 1060 . The value of $\delta$, the boundary layer thickness, in the region of the measurement planes was $69 \mathrm{~mm}$.

The flow was seeded with olive oil droplets (size $\sim 1 \mu \mathrm{m}$ ) that were generated by eight Laskin nozzle units set up in parallel. The oil droplets were ingested into the intake of the wind tunnel upstream of honeycomb straighteners and screens used for flow conditioning. At the test section, glass side-walls and a glass bottom wall were installed in the wind tunnel for optical 


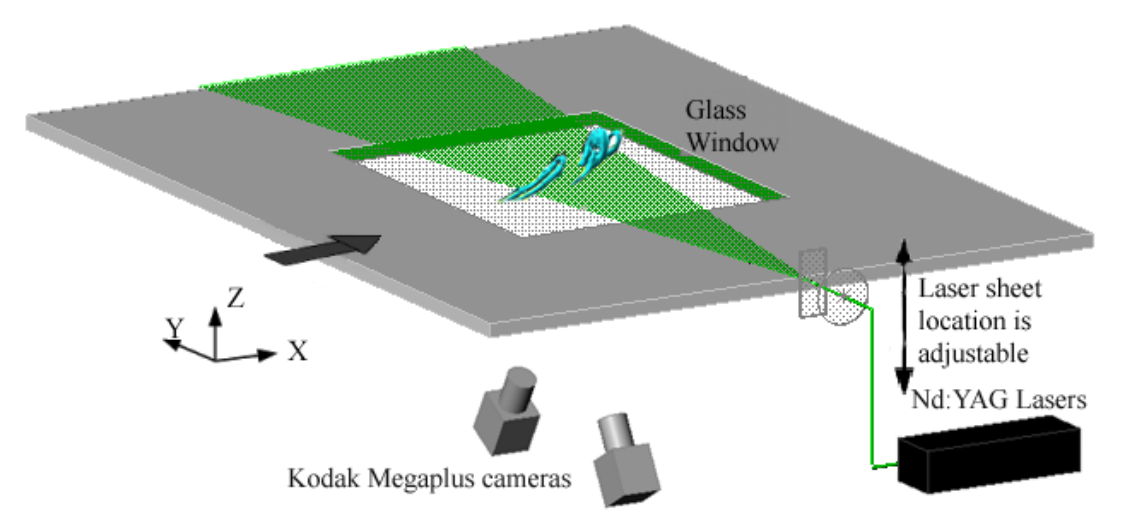

Figure 1. Experimental facility from [23]

access. The seed particles were illuminated by pulsed sheets from a pair of Nd:YAG lasers (Big Sky CFR200) directed through one side window and oriented parallel with the bottom wall as shown in figure 1. The laser pulse energy was $120 \mathrm{~mJ}$, and the thickness of each sheet was about $0.4 \mathrm{~mm}$. Sets of digital images were captured at a frequency of $15 \mathrm{~Hz}$ by two Kodak Megaplus CCD cameras $(1024 \times 1024$ pixels $)$ at $z^{+}=92(0.085 \delta)$. Nikon Micro Nikkor $60 \mathrm{~mm} \mathrm{f} / 2.8$ lenses were used with the cameras. A TSI synchronizer box was used to control the strobing and timing of the cameras and lasers. The cameras were aligned in a plane parallel with the $x-y$ flow plane and inclined at angles of $15^{\circ}$ with the $z$ axis as shown in figure 1 . Additional details of the calibration, experimental arrangement and vector reconstruction schemes are given in [24].

For all derived quantities, the interrogation spot size used was $16 \times 16$ pixels $(\sim 20 \times 20$ wall units) with $50 \%$ overlap. The spacing between adjacent vectors in either direction is 10 wall units $(\sim 0.65 \mathrm{~mm})$. The measurements had two main sources of uncertainty. First, the Gaussian peak fit in the cross-correlation algorithm generated an uncertainty of approximately 0.1 pixels or $0.016 \bar{U}$ (where $\bar{U}$ is the mean velocity in the streamwise direction). Second, a residual error arose due to the least-square curve-fit in solving the four pixel-displacement equations in three unknowns (see [24] for details). This residual error can play a major role in the uncertainty if there are concentration gradients in seeding within the flow field. In this experiment, such gradients were not significant, and this error was on average about half of the Gaussian error. The vector fields from the images acquired with the Megaplus cameras covered an area of $1.2 \delta \times 1.2 \delta$.

Velocity gradients were computed from vector fields using a second order central difference scheme wherever possible in the domain and a first order forward or backward difference at the boundaries. To identify swirling motion caused by the eddies in the flow field, we used swirl strength $\lambda_{c i}$ [19], which is defined as the magnitude of the imaginary part of the eigenvalue of the local velocity gradient tensor. Since the PIV images were planar, only the in-plane gradients were used to form a two dimensional tensor. Vortices could be identified by extracting iso-regions of $\lambda_{c i}$ as suggested by Adrian et al [25].

\section{Visualization of stereo PIV datasets}

In order to demonstrate visualization strategies, we have chosen a single representative vector field from the stereo PIV results. In fact, many vector fields were examined with each of these strategies. 


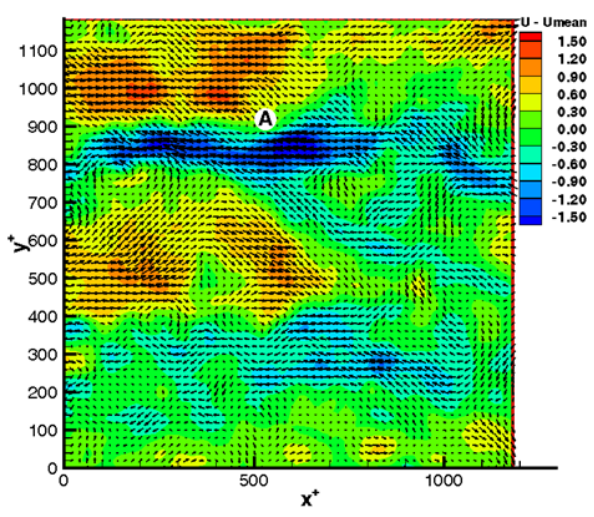

(a)

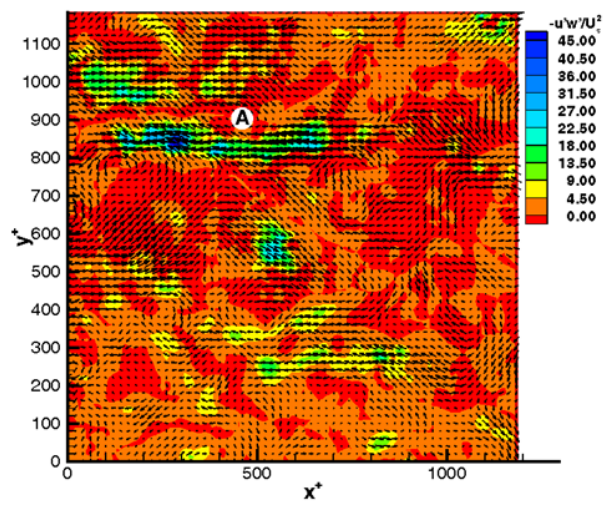

(c)

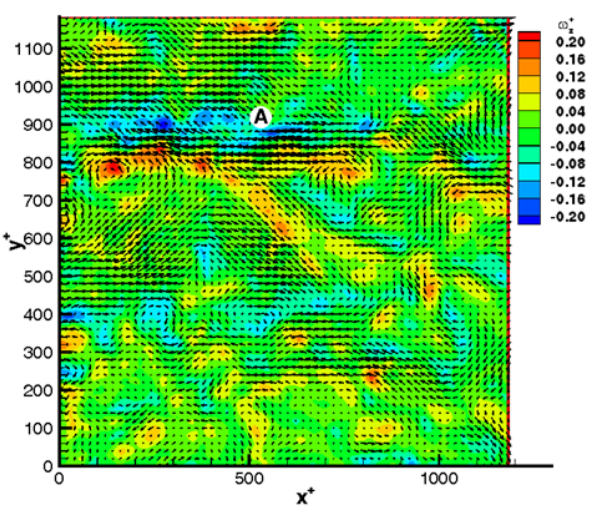

(b)

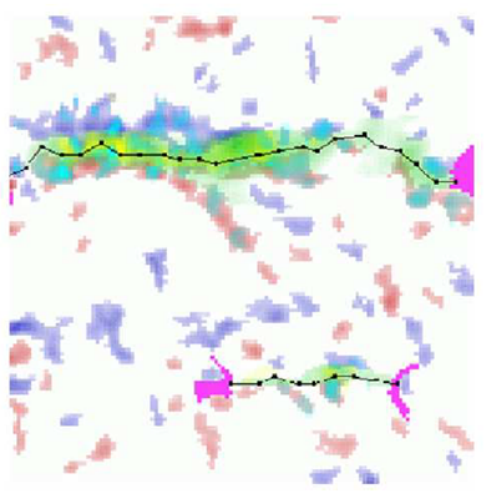

(d)

Figure 2. (a) $U-\bar{U}$, (b) wall-normal vorticity $\left(\omega_{z}\right)$, (c) Reynolds shear stress $-u w^{+}$, (d) four parameters in one image. Red and blue show vorticity, green shows streamwise velocity only in the region of the hairpin packet found by the feature extraction algorithm and cyan and yellow show swirl strength and Reynolds shear stress respectively.

\subsection{D colour plots}

Various quantities can be visualized in order to investigate the nature of the boundary layer flow. In the current study, four quantities of primary importance were chosen: instantaneous Reynolds shear stress $(-u w)$, wall-normal vorticity $\left(\omega_{z}\right)$, swirl strength $\left(\lambda_{c i}\right)$ and streamwise velocity $(U)$. These quantities can all be employed to help identify and characterize vortex packets that may exist in the log region. As explained in [23], vortex packet signatures at the wall-normal position depicted herein $\left(z^{+}=92\right)$ contain streamwise trains of hairpin vortex legs characterized by discrete regions of strong swirl embedded in streamwise strips of opposing vorticity. The strips of strong vorticity surround zones with relatively uniform streamwise velocity that contain intermittent spots of large Reynolds shear stress. The packet structures and their relative frequency of occurrence at various wall-normal positions are described in detail in [23]. The typical size and spacing of the dominant flow structures are further quantified in [26] using two-point correlations and additional analysis.

Figures 2(a)-(c) depict streamwise velocity, vorticity and Reynolds shear stress respectively using colour contours. The region marked (A) in all three plots has features indicative of a 
vortex packet. Figure 2(a) depicts an elongated region of near constant (and relatively small) streamwise velocity in blue. The Reynolds shear stress is quite high throughout this region as seen in figure 2(c), and, furthermore, the region is bounded at the top and bottom by zones of negative and positive vorticity (figure 2(b)). (A separate plot of swirl strength indicates that the vortical strips contain distinct vortex cores.)

In the previous study [23], we used this combination of characteristics to develop an automated feature extraction algorithm. To identify a packet visually using the colour schemes shown, however, it is necessary to look at three or more separate plots of a given data field. From a visualization standpoint, this is quite a tedious process. Hence, we developed a tool in which four primary variables could be viewed in the same image. As the tool was designed to highlight hairpin vortex packets visually, it requires the use of the feature extraction algorithm as well as thresholding techniques.

In figure $2(\mathrm{~d})$, red and blue show regions of positive and negative vorticity with magnitudes greater than $1.5 \sigma_{\omega_{z}}^{+}$where $\sigma_{\omega_{z}}^{+}$is the root mean square of $\omega_{z}^{+}$. Yellow depicts zones of strong Reynolds stress occurring between the red and blue regions. Green shows regions of relatively uniform streamwise velocity that are activated only in regions identified as hairpin packets by the algorithm. The upper threshold for a green region is the average streamwise velocity in the neighbouring vorticity regions, and the lower threshold is the minimum velocity in the yellow Reynolds shear stress zones. Cyan depicts zones of strong swirl that occur within packets. In general, stronger hues indicate larger magnitudes.

Two other features are depicted in figure 2(d). First, black curves mark the spanwise centre of each hairpin packet identified. This is useful for measuring statistics on packet length and width. Second, pink regions mark search zones upstream and downstream of each packet. Individual packets identified by the feature extraction algorithm can be merged provided that the streamwise distance between them is less than the average width of the patches being merged. The pink areas thus depict the area searched for adjacent packets.

The composite plot is fairly effective at highlighting the packet described above in that all four primary variables and the relationship between them can be observed. Also, the plot reveals and helps characterize a second shorter packet identified by the algorithm. This packet would have been nearly impossible to identify visually from the separate colour plots in figures 2(a)-(c). The composite plot does have shortcomings however. First, it is impossible to view the extent of overlap of the different variables (or features) plotted. We cannot tell from the plot, for example, whether or to what extent parts of swirling zones also contain strong Reynolds stress. Second, the green regions in the plot are difficult to interpret. They coincide frequently with yellow and cyan zones, and otherwise give no indication of the velocity magnitude within them. These problems can be alleviated by incorporating visualization techniques not dependent on colour.

\subsection{Line integral convolution of vector fields}

A standard method to visualize velocity magnitude and direction in planar fields is a vector plot formed by a grid of arrows. This method can be fairly effective at depicting variations of in-plane velocity components. However, it does have some limitations. For example, if the vector spacing is too dense, individual vectors can overlap, obscuring the details of velocity variations. Also, structural features of the flow such as strongly swirling or straining regions are not always easy to perceive. Figure $3(\mathrm{a})$ is a vector plot of the velocity field with mean velocity $(\bar{U})$ subtracted in order to accentuate between relatively fast and slow moving zones. The region marked (A) is the same slow-moving region shown in figure 2(a). The vector field gives us the idea that this region has relatively low velocity, but the velocity magnitude is much easier to determine from the colour contours in figure 2(a). The spot marked (B) is surrounded by several vortical structures, but they are not particularly well resolved. 


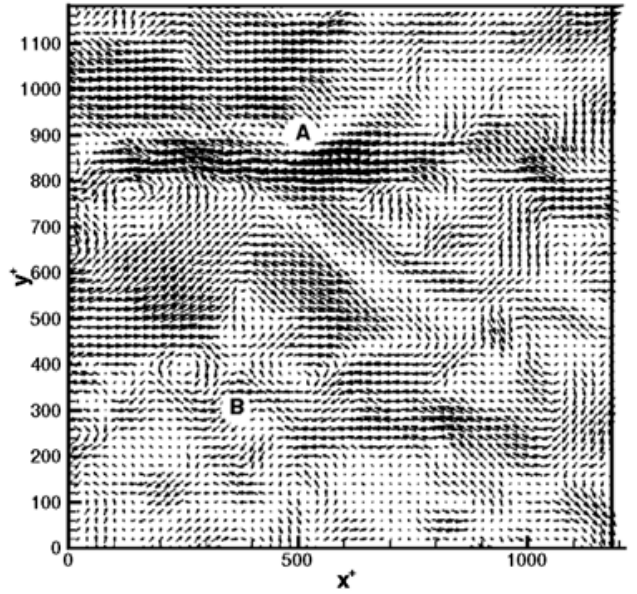

(a)

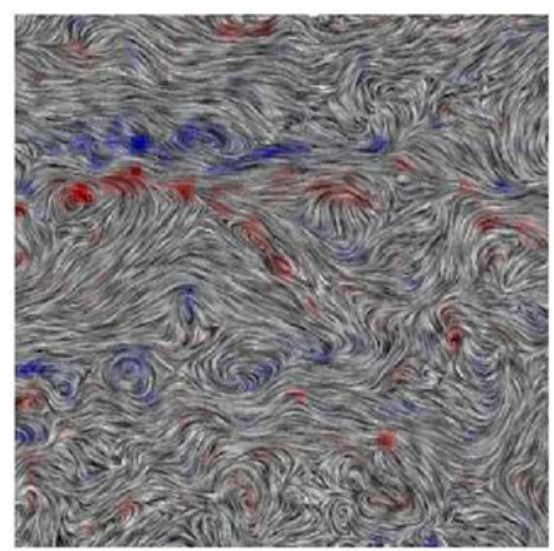

(c)

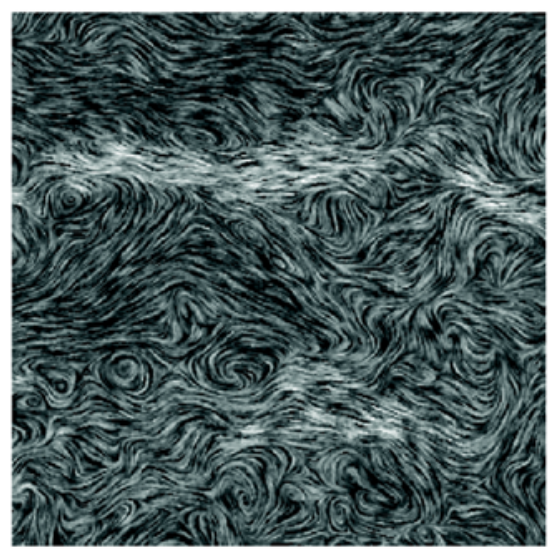

(e)

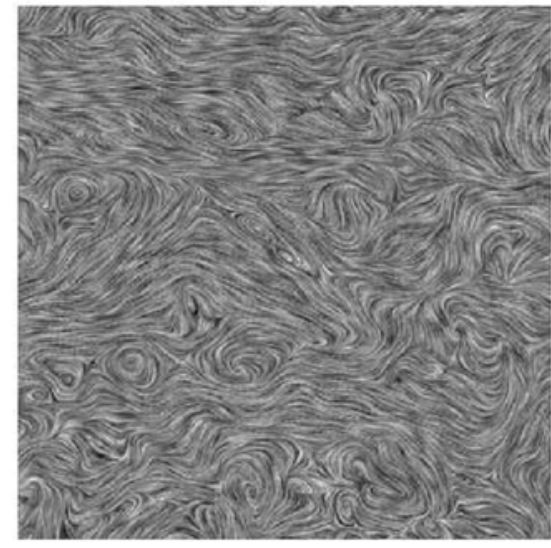

(b)

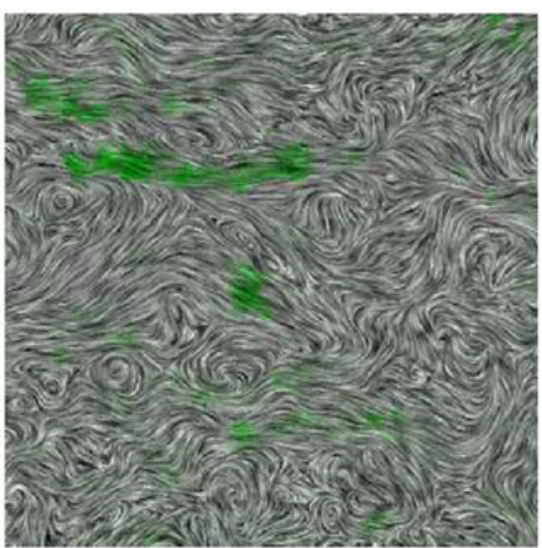

(d)

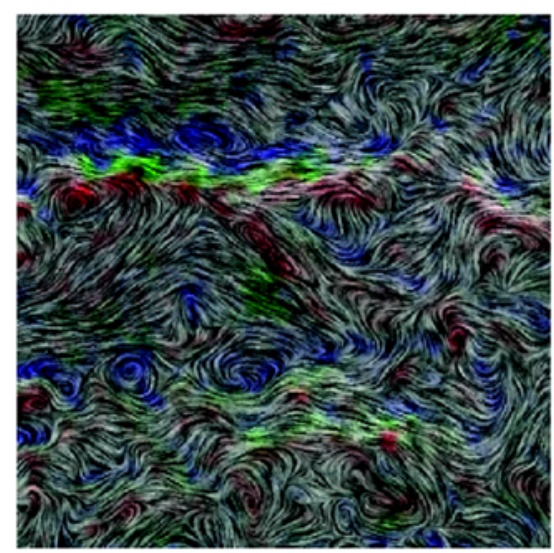

(f)

Figure 3. All vectors and streamlines are plotted using $U-\bar{U}$ and $V$. (a) Vector plot, (b) line integral convolution (LIC) static image, (c) LIC with $\omega_{z}$, red-positive vorticity, blue - negative vorticity, (d) LIC with $-u w^{+}$as green colour contours, (e) LIC with contrast depicting low momentum regions, (f) LIC base image with $\omega_{z},-u w^{+}$and momentum in colours. 
The features in the vector plot can be depicted with finer resolution and greater clarity using a texture-based method such as LIC. With this method, an input texture, such as white noise, is convolved along streamlines calculated from the vector field. The method, first introduced by Cabral and Leedom in 1993 [27], yields an output image in which instantaneous streamlines appear as continuously varying textured curves.

We employed the fast LIC algorithm of Stalling and Hege [28] to generate textured fields. First, streamlines were calculated from the points on the vector grid, where a fourthorder Runge-Kutta scheme determined incremental positions along the calculated streamline. Adaptive step sizes were employed to speed up the computation, while error monitoring limited step sizes to ensure high accuracy. Once the streamlines were calculated, they were re-sampled at equally spaced points for use in sampling the input texture. Each pixel in the final image was determined by averaging $2 N$ pixels of the input texture along the streamline section centred on that pixel. The resulting image has textured patterns depicting the streamline orientation throughout the field. In addition to streamline orientation, LIC images can also depict vector magnitude by varying the kernel length $N$ according to the magnitude [29]. This results in longer textures corresponding to vectors of higher magnitude.

In the images shown, the kernel length nominally ranges from 5 to 14 pixels. If this range were increased, for example, regions of larger magnitude would appear coherent over longer distances than seen in the present image. Also, the zones with very small velocity magnitude would appear short and incoherent.

The LIC-generated image (figure 3(b)) depicts the low momentum zone in region A by horizontally oriented textures. Similar textures appear also near the top of the image and through region $\mathrm{B}$, although in these areas the streamwise velocities are not as low as region A. The local streamline orientation is also easy to see. (Note, for example, the spanwise motion between A and B as well as the swirling zones near B that are also observable in the vector plot.) The appearance of these swirling zones (as well as the general streamline orientation) depends, however, on the specific choice of streamwise velocity in the input vector field $\left(U-U_{c}, V\right)$. In this plot, $U_{c}=\bar{U}$ where $U_{c}$ is a convection velocity. If a different streamwise reference frame were chosen by altering $U_{c}$, the swirling zones would disappear. Hence some iteration may be required to find the optimal value of $U_{c}$ to match zones of high swirl $\left(\lambda_{c i}\right)$ to swirling LIC patterns.

The LIC image becomes more useful when other derived parameters are overlaid with colours. Figures 3(c) and (d) show the LIC image overlaid with red and blue for strong vorticity and green for strong Reynolds stress. In this way, it is easy to determine the velocity orientation (in the given reference frame) corresponding with the coloured quantities. An additional technique can be applied to emphasize the low velocity regions. In figure 3(e), regions with low streamwise velocity are highlighted by using visual contrast. These regions are depicted by increasing the brightness when the streamwise velocity falls below a threshold value. In figure 3(f), all possible features shown in figures 3(b)-(e) are represented in one image. One issue encountered is the effect of superposition of multiple colours on the appearance of the final image.

The manner in which the colours are composited is very important. For example, one possible method of compositing the colours is to set the colour of the final image to the colour of the layer added most recently. Another possible method is to set the colour of the final image as the sum of all colours at that location. This method was rejected because it resulted in generation of new colours in overlap regions that had no obvious meaning. In figure 3(f) the colour of the final image was set to the product of the colours in the layers, which makes the region of overlap darker but does not change the colour scheme. Thus, using the product function is similar to overlaying semi-transparent colour filters. This method seems to work well when two colours overlap, which is the maximum number possible in figure $3(\mathrm{f})$, but might present problems when three or more colours overlap. 


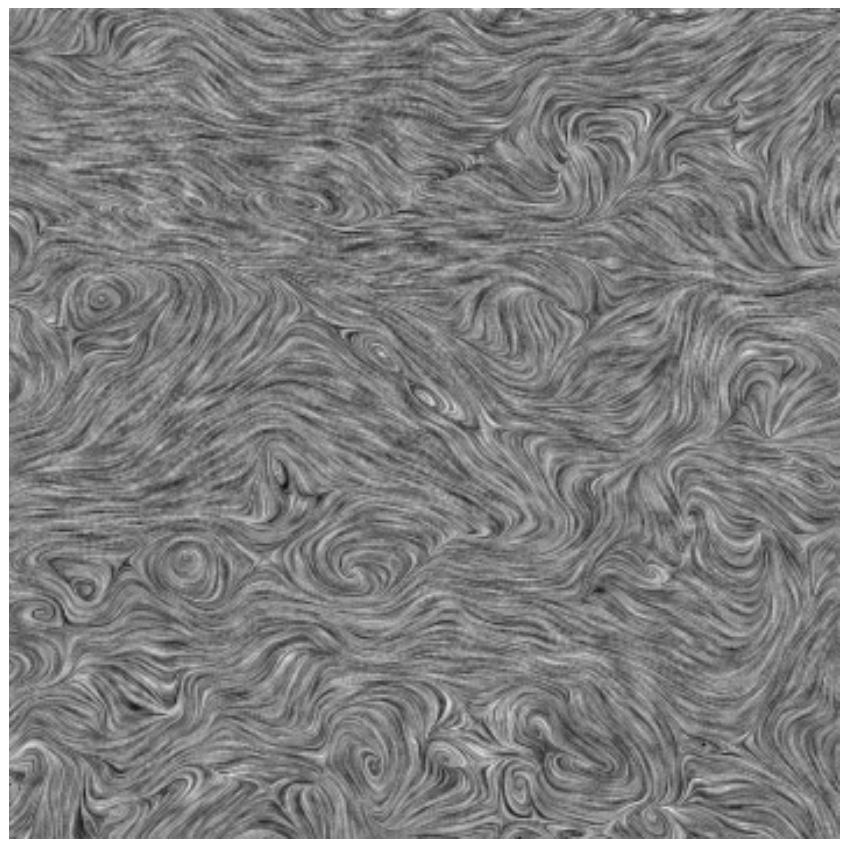

Figure 4. LIC animation of one $(U-\bar{U}, V)$ field.

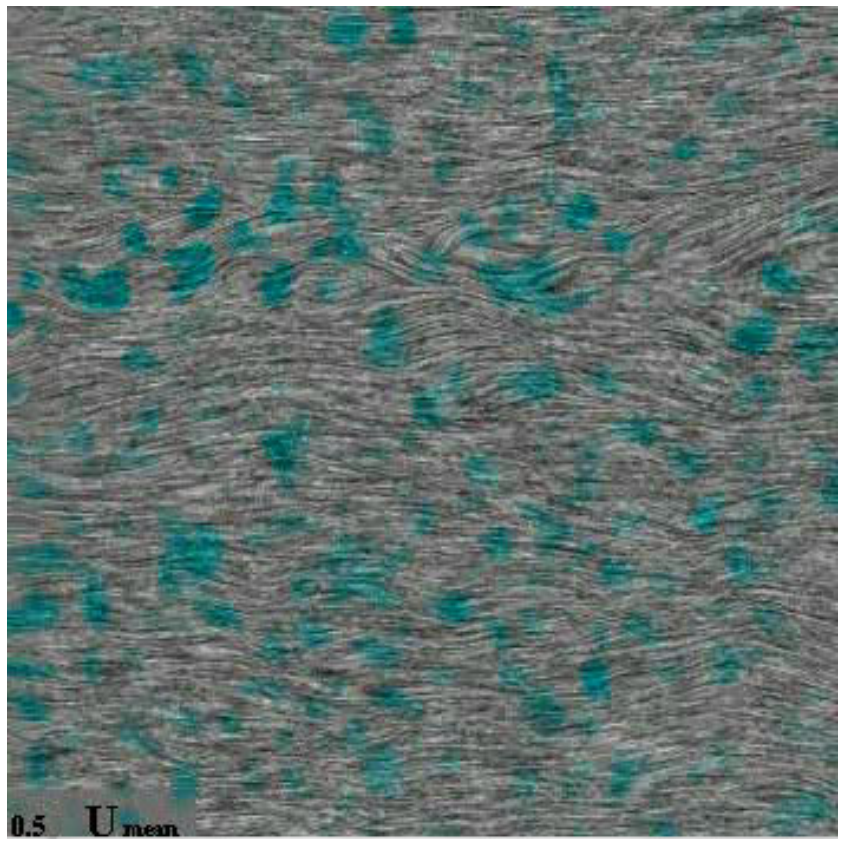

Figure 5. LIC animation of $\left(U-U_{c}, V\right)$ field for varying values of $U_{c}$ (displayed in bottom left corner). Cyan overlay corresponds to swirl strength.

\subsection{Animations with 2D LIC images}

Even though the combination of LIC and colour gives a better visualization of the flow structure than the simple use of colours shown in figure 2(d), it leaves room for improvement. The static LIC images show streamline orientation well, but they do not reveal the flow direction (e.g. left versus right for a horizontally oriented streamline). One way to provide more information 
(and clarification) to the viewer is to animate the static LIC field. The standard LIC image uses an equal length kernel filter in both directions along the streamline centred at the pixel being rendered. By shifting the kernel in the direction of the flow, a second LIC image can be generated that is highly correlated to the first, but with the pixel values altered in correspondence with the flow direction. By performing this kernel shift over several time steps and windowing appropriately (see [28]), an animation can be generated that depicts the instantaneous flow direction corresponding with the original PIV field. The velocity magnitude can also be represented in the animation by scaling the kernel shift to the local magnitude. Thus, areas with high magnitude vectors appear to travel faster than areas with low magnitude vectors. An animated GIF file generated using the $(U-\bar{U}, V)$ vector field (see animation, figure 4 ) is provided. The file loops through a set of six images generated using the process described above.

The animation shown thus provides the direction and relative magnitude of the instantaneous local velocity in the given reference frame. It is important to note that, because the data represent the velocity field at only one instant in time, local acceleration terms are unknown, and the animated vector field does not represent a true picture of the evolving motion. Nevertheless, the animation emphasizes the locations of low and high speed flow, swirling regions, and converging and diverging flow at this instant in time. As in the static LIC field in figure 2(d), we must note also that the streamlines and flow patterns shown depend explicitly on the reference frame chosen. The swirling eddies near the bottom left of the flow field are evident only because we have chosen a frame convecting at $\bar{U}$ which happens to match the convection velocity of those eddies.

Since the swirl strength $\lambda_{c i}$ is Galilean invariant, it can be used in combination with the LIC animations to determine the convection velocities of various eddies in the flow. Specifically, the swirl strength can be overlaid on LIC animations computed using a range of values $U-U_{c}$ to determine under what conditions locations with large $\lambda_{c i}$ coincide with swirling streamlines. The file (see animation figure 5) contains a series of LIC animations in which $U_{c}$ was varied from $0.5 \bar{U}$ to $1.5 \bar{U}$. By stepping through the various animations, it becomes apparent that swirling streamlines appear and disappear at different values of the convection velocity. In addition, many other specific features are emphasized at distinct convection velocities. For example, the long hairpin packet designated by $\mathrm{A}$ in the earlier figures is seen to convect in the range $0.85 \bar{U}-1.05 \bar{U}$. Also, many of the swirling zones appear to convect at the streamwise local mean velocity $(\bar{U})$.

\section{Multi-dimensional visualization}

So far we have seen that the texture-based LIC method can be used in combination with colour overlays to represent multiple flow parameters effectively. As stated earlier, however, the number of colours that can be overlapped without ambiguity is limited. In order to incorporate additional parameters into a single visualization, a third dimension can be employed. Height can be useful to discriminate spatially discrete zones of one parameter that may overlap with or bound another. However, the choice of an appropriate and suitable variable is necessary for the use of height. Because swirl strength is a scalar limited to positive values, and because high swirl strength typically appears in discrete circular areas that lie within zones of significant vorticity, we chose to represent it with height in a three-dimensional plot. Figure 6(a) shows a plot of an LIC field generated from $(U-\bar{U}, V)$ with the third dimension used to represent swirl values. Figure $6(\mathrm{~b})$ shows the same parameters with colours and brightness overlaid to represent additional features. As in figure 3(f), shades of green correspond to regions of high Reynolds shear stress, and blue and red correspond to negative and positive zones of wall-normal vorticity. Brightness is used to emphasize the coherent regions of low streamwise velocity identified by the feature extraction algorithm. 


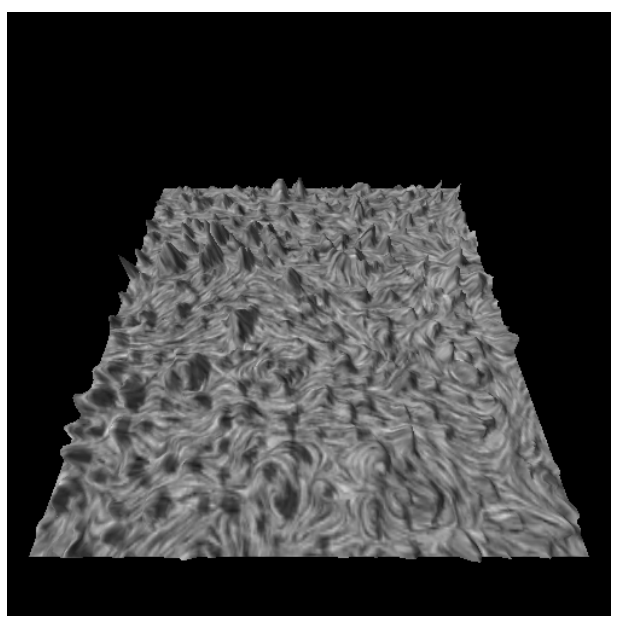

(a)

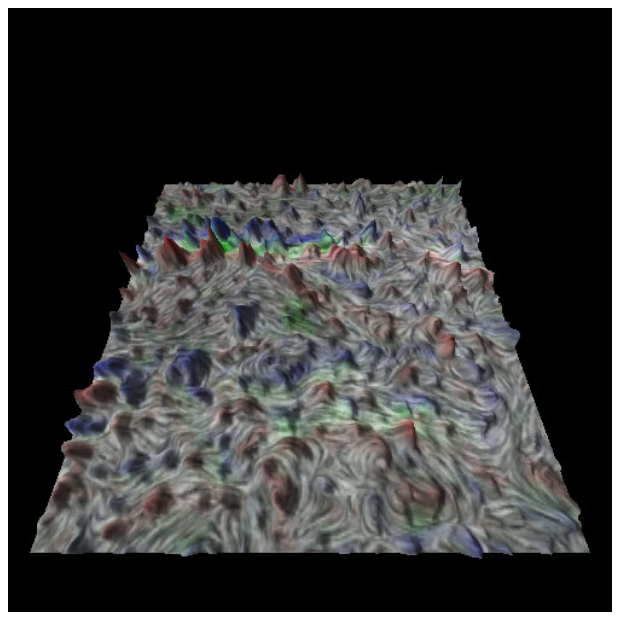

(b)

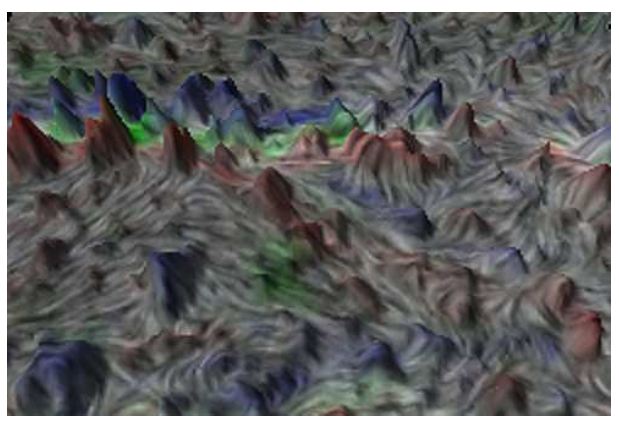

(c)

Figure 6. (a) LIC-based vector field with swirls accentuated as peaks, (b) the same as (a) with colours. Green represents $-u w^{+}$and blue and red elucidate positive and negative $\omega_{z}{ }^{+}$. The contrast represents the low momentum zones. (c) A blow-up of the vortex packet at the top of the image in figure 6(b).

A different perspective of the same data (a blow-up of the hairpin packet at the top of the field) is shown in figure 6(c). This image, which is equivalent to focusing on the hairpin packet with a zoom lens rather than a wide-angled lens, gives a better idea of the interaction between height and LIC textures. Different perspectives can therefore be used to emphasize or view the interaction between 'plotted' variables.

The use of the third dimension to represent swirl accentuates its discrete zones. It also shows effectively that the discrete swirl zones (associated with vortices) can be embedded within longer continuous zones of strong vorticity as occurs in the long hairpin packet. Also, the use of height for swirl helps emphasize that these zones can surround streamwise streaks of low momentum fluid with significant Reynolds stress. The three-dimensional representation can also be animated and viewed from different angles (see animation, figure 7 ). In the three-dimensional animation, the hairpin packet appears as a river running between two mountain ranges.

\section{Summary and conclusions}

In this paper, we have attempted to demonstrate methods in which the flow structure described by planar fields of three-dimensional velocity vectors can be visualized effectively in a single plot. In addition to local velocity values, the fields also contain information on in-plane 


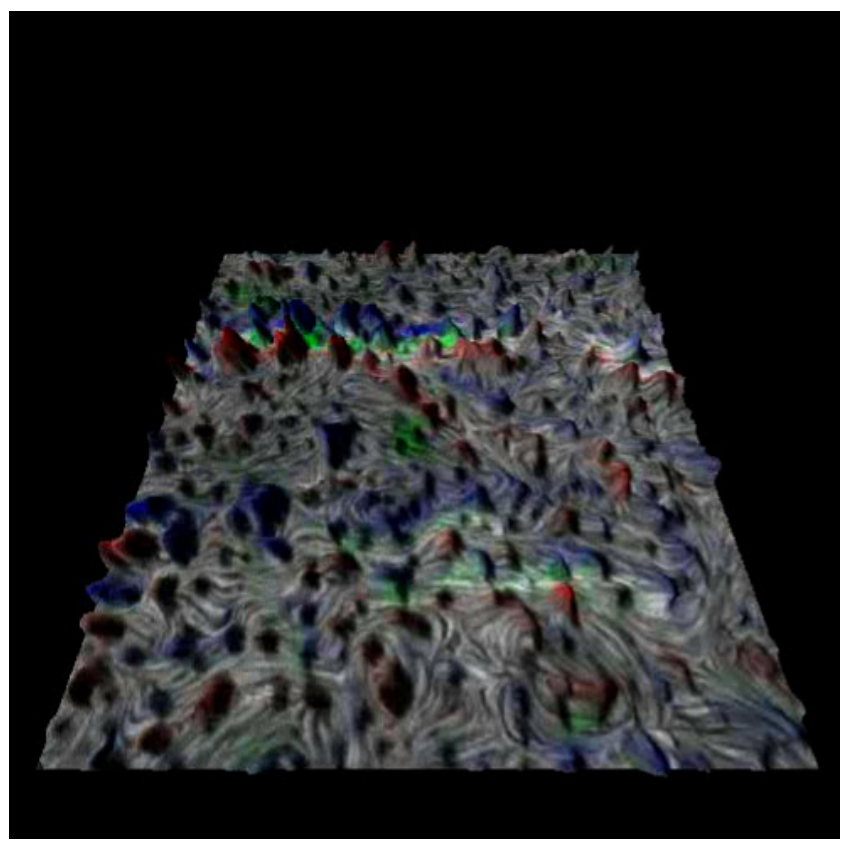

Figure 7. Animation showing different viewing angles of image in figure 6(b).

velocity gradients. For the turbulent boundary layer examined, vorticity, swirl strength, instantaneous Reynolds shear stress $(-u w)$ and streamwise velocity were considered as useful quantities to visualize because, in combination, they could be used to identify and characterize packets of hairpin vortices.

Several methods for simultaneous visualization of multiple quantities were demonstrated. A critical issue concerns effective visualization of regions where several quantities of interest overlap. A method using colour alone was observed to have limitations with regard to this issue. Although two overlapping colours can be composited effectively, if more than two overlap, some information will become ambiguous or obscured or at least difficult to interpret.

It was shown that texture could be used instead of a standard vector grid to visualize the velocity field effectively. An animated LIC method using a white noise input texture was effective at showing local variations in velocity magnitude and direction. In addition, this method could be employed in combination with translucent colour overlays so that out-of-plane quantities $(-u w)$ or derived quantities (vorticity) could be related to the in-plane velocity field. The LIC method applied to different translation frames was also useful for determining the convection velocity of specific structures, e.g. swirling zones.

Three-dimensional visualization provided an additional tool for distinguishing one scalar variable from other potentially overlapping ones represented using colour. In this paper, height was used to plot local swirl values. This parameter was chosen (as opposed to vorticity or Reynolds stress) because of the typical size and shape of high swirl strength regions. These regions tended to be smaller than regions of high vorticity with which they frequently overlapped. Also the coincidence of high swirl zones with vortex cores yielded swirling peaks in the threedimensional animations that could easily be associated with (or perceived as) vortices.

In the current study, the choice of colour, brightness and height to represent specific parameters was guided by the typical flow structures present in the log region of the boundary layer flow. In other regions of this flow, or in other flow geometries, other combinations might prove superior. Also, the current study represents only one demonstration of combining texture, brightness, height and colour contours to view multiple quantities in a single plot. 
The current technique might be improved in the future through additional study on details of the texture-based method as well as on possibilities for combination of colours and threedimensionality in overlapping regions. The LIC method shown in this paper could be further optimized through study of the effect of kernel length and background texture on viewer perception. In addition, other texture-based methods could be examined (see [30,31]). In future experiments, we plan to obtain dual-plane stereo-PIV measurements that will allow determination of all nine spatial velocity gradients within a planar grid. In this case, it will be possible, for example, to determine the full vorticity vector. As the vorticity vector orientation is critical to understanding the eddy structure in turbulence, a method of displaying this quantity in combination with some of the others listed above would be extremely desirable.

\section{Acknowledgments}

The authors wish to thank Margaret Richey, Noor Martin and Kirti Kesavarapu for being instrumental in generating the images in this paper. This work was supported by the National Science Foundation through grants ACI-9982274 and CTS-9983933.

\section{References}

[1] Hama F R, Long J D and Hegarty J C 1956 On transition to laminar to turbulent flow J. Appl. Phys. 284

[2] Corrsin S 1957 Some current problems in turbulent shear flows Symp. Naval Hydrodynamics (Washington, DC, Sept. 1956) National Academy of Science-National Research Council Publication 515

[3] Kline S J and Runstadler P W 1959 Some preliminary results of visual studies of the flow model of the wall layers of the turbulent boundary layers Trans. ASME E 2 166-70

[4] Corino E R and Brodkey R S 1969 A visual investigation of the wall region in turbulent flow J. Fluid Mech. 37 $1-30$

[5] Kline S J, Reynolds W C, Schraub F A and Runstadler P W 1967 The structure of turbulent boundary layers J. Fluid Mech. 30 741-73

[6] Cantwell B J 1981 Organized motion in turbulent flow Annu. Rev. Fluid Mech. 13 487-515

[7] Robinson S K 1991 Coherent motions in turbulent boundary layers Annu. Rev. Fluid Mech. 23 601-30

[8] Panton R L 1997 Self-Sustaining Mechanisms of Wall Turbulence (Southampton, UK: Comp. Mech. Publications)

[9] Jeong J, Hussain F, Schoppa W and Kim J 1997 Coherent structures near the wall in a turbulent channel flow J. Fluid Mech. 332 185-214

[10] Schoppa W and Hussain F 2002 Coherent structure generation in near-wall turbulence J. Fluid Mech. 453 $57-108$

[11] Heist D K, Hanratty T J and Na Y 2000 Observations of the formation of streamwise vortices by rotation of arch vortices Phys. Fluids 12 2965-75

[12] Theodorsen T 1952 Mechanism of turbulence Proc. 2nd Midwestern Conf. on Fluid Mech. (Columbus, OH)

[13] Head M R and Bandyopadhyay P 1981 New aspects of turbulent boundary-layer structure J. Fluid Mech. 107 297-338

[14] Perry A E and Chong M S 1982 On the mechanism of wall turbulence J. Fluid Mech. 119 173-217

[15] Townsend A A 1976 The Structure of Turbulent Shear Flow (Cambridge: Cambridge University Press)

[16] Perry A E and Marusic I 1995 A wall-wake model for the turbulence structure of boundary layers. Part 1. Extension of the attached eddy hypothesis J. Fluid Mech 298 361-88

[17] Adrian R J, Meinhart C D and Tomkins C D 2000 Vortex organization in the outer region of the turbulent boundary layer J. Fluid Mech. 422 1-54

[18] Tomkins C D and Adrian R J 2002 Spanwise structure and scale growth in turbulent boundary layers TAM Reports University of Illinois at Urbana-Champaign no 1006

[19] Zhou J, Adrian R J, Balachandar R J and Kendall T M 1999 Mechanisms for generating coherent packets of hairpin vortices in channel flow J. Fluid Mech. 387 353-96

[20] Adrian R J and Liu Z C 2002 Observation of vortex packets in direct numerical simulation of fully turbulent channel flow J. Vis. 5

[21] Christensen K T and Adrian R J 2001 Statistical evidence of hairpin vortex packets in wall turbulence J. Fluid Mech. 431 433-43 
[22] Marusic I 2001 On the role of large-scale structures in wall turbulence Phys. Fluids 13 735-43

[23] Ganapathisubramani B, Longmire E K and Marusic I 2003 Characteristics of vortex packets in a turbulent boundary layer J. Fluid Mech. 478 35-46

[24] Ganapathisubramani B, Longmire E K and Marusic I 2002 Investigation of three dimensionality in the near field of a round jet using stereoscopic PIV J. Turbulence 3016

[25] Adrian R J, Christensen K T and Liu Z C 2000 Analysis and interpretation of instantaneous turbulent fields Exp. Fluids $29275-90$

[26] Longmire E K, Ganapathisubramani B and Marusic I 2003 Analysis of vortex packets and Reynolds stress in a turbulent boundary layer Proc. 3rd Int. Symp. on Turbulence and Shear Flow Phenomena (Sendai, June 2003) to be published

[27] Cabral B and Leedom L 1993 Imaging vector fields using the line integral convolution Computer Graphics Proc. (Annu. Conf. Series) pp 601-39

[28] Stalling D and Hege H C 1995 Fast and resolution independent line integral convolution ACM SIGGRAPH'95 Conf. Proc. (Annu. Conf. Series) pp 249-56

[29] Kiu M-H and Banks D C 1996 Multi-frequency noise textures for LIC Proc. IEEE Visualization 96 (San Francisco, CA) (Piscataway, NJ: IEEE) pp 121-6

[30] Kirby R M, Marmanis H and Laidlaw D H 1999 Visualizing multivalued data from 2D incompressible flows using concepts from painting Proc. IEEE Visualization (Piscataway, NJ: IEEE) pp 333-40

[31] Healey C G and Enns J T 1999 Large datasets at a glance: combining textures and colors in scientific visualization IEEE Trans. Vis. Comput. Graphics 5 145-67 